\title{
Review-electrochemical detection of uric acid, dopamine and ascorbic acid
}

\begin{abstract}
Uric acid (UA), dopamine (DA) and ascorbic acid (AA) exist in the human body as protein metabolism, neurotransmitter and antioxidant, respectively. The abnormal levels of UA, DA and AA could lead to certain diseases and it could be measured and determined using electrochemical sensors. UA, DA and AA have close oxidation potentials and cause overlapping peaks in electrochemical measurements. Researchers have been working on electrode modification by using different materials to overcome those challenges and improve their selectivity, sensitivity and limit of detection. This review aims to highlight the performance of modified electrochemical sensors for detection of UA, DA and AA individually or simultaneously.
\end{abstract}

